\title{
On-line Breath Metabolomics in Respiratory Diseases using Secondary Electrospray Ionization-Mass Spectrometry
}

\author{
Martin Thomas Gaugg* \\ SCS-Metrohm Award for best oral presentation in Analytical Sciences
}

\begin{abstract}
Every second we are exhaling hundreds of endogenous and exogenous compounds that originate from blood and lung tissue. Obtaining metabolic information via exhaled breath analysis has been an emerging topic since the 1970s. Secondary electrospray ionization-mass spectrometry is a relatively new technique to detect these metabolites on-line in a highly sensitive and specific fashion. Using this technique, several respiratory diseases, including chronic obstructive pulmonary disease, obstructive sleep apnea, idiopathic pulmonary fibrosis, asthma, and lung cancer have been investigated over the past years. Several new potential biomarkers for these diseases were identified and new metabolic insights into their pathophysiology could be obtained.
\end{abstract}

Keywords: Breath analysis · Mass spectrometry $\cdot$ Respiratory diseases $\cdot$ Secondary electrospray ionization

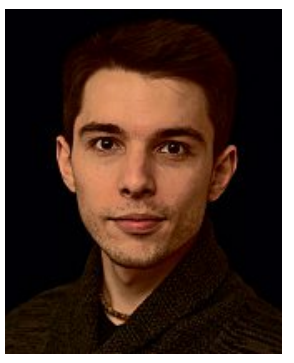

Martin T. Gaugg was awarded his Bachelor's degree in Chemistry at Leopold-Franzens-University Innsbruck in 2013 as best of his year and continued his education at ETH Zürich, where he completed his Master's degree in chemistry under the Excellence Scholarship and Opportunity Programme (ETH Zürich) in 2015. Already during his studies, Martin joined the group of Prof. Renato Zenobi, where he developed automated dataprocessing algorithms for on-line breath analysis, and is currently pursuing his $\mathrm{PhD}$ studies there in a collaboration with the University Hospital Zürich, investigating new biomarkers for respiratory diseases using breath analysis.

\section{Introduction}

Respiratory diseases are amongst the most common causes of death worldwide and contribute significantly to public health costs. ${ }^{[1]}$ Early diagnosis and treatment is crucial for improved patient outcome and to reduce unnecessary costs. Unfortunately, most tests available at the moment either give purely physical information about the patient's lung capacity (lung function test), providing no objective measure based on biochemical information, are highly invasive (lung biopsies) or expose the patient to high doses of radiation (chest X-ray or CT). This results in tardy diagnosis and treatment, and a poor understanding of those diseases. Hence, non-invasive diagnostic tools that can simultaneously give information about the underlying metabolism would be of tremendous value.

Our breath contains hundreds of volatile organic compounds, including metabolites, hormones, inhaled exogenous substances and many more. ${ }^{[2]}$ Our breath therefore contains highly valuable information about our body, including diseases, ${ }^{[3]}$ metabolic processes ${ }^{[4]}$ as well as unique patterns for every person. ${ }^{[5]}$ Since Linus Pauling first reported over 200 compounds in exhaled breath in the 1970s, [6] over 800 endogenous and exogenous metabolites have been identified in exhaled breath of humans. ${ }^{[7]}$ It is well known that animals with an excellent sense of smell (e.g. dogs) can identify certain diseases simply by sniffing a patient's odor. ${ }^{[8]}$ It is therefore reasonable to assume that lung diseases in particular have a specific impact on the pattern of exhaled compounds. Detection of these diagnostic features in a standardized fashion would open the door for quick and non-invasive diagnosis of a variety of diseases. ${ }^{[3 b]}$ Several analytical techniques have been developed in order to achieve this goal, including off-line and on-line methods. The gold-standard for off-line analysis of breath is gas chromatography-mass spectrometry, which allows for absolute quantification by addition of internal standards. However, it requires several sample handling steps (collection in bags, pre-concentration on adsorbents, etc.) which can compromise the sample and prolongs the analysis process. ${ }^{[9]}$ Therefore, several groups are focused on developing techniques to analyze exhaled breath in real-time without any sample preparation (Fig. 1).

Until recently, the most prominent methods for this purpose were proton transfer reaction-mass spectrometry (PTR-MS) ${ }^{[10]}$ and selected ion flow tubemass spectrometry (SIFT-MS). [11] In 2007, Sinues and co-workers found that secondary electrospray ionization-mass spectrometry (SESI-MS) is a highly sensitive and soft ionization technique to not only ionize breath aerosols, but also breath metabolite vapors directly at ambient pressure. ${ }^{[12]}$

Our group therefore focuses on further developing SESI-MS, and using it to identify new biomarkers for respiratory dis- 


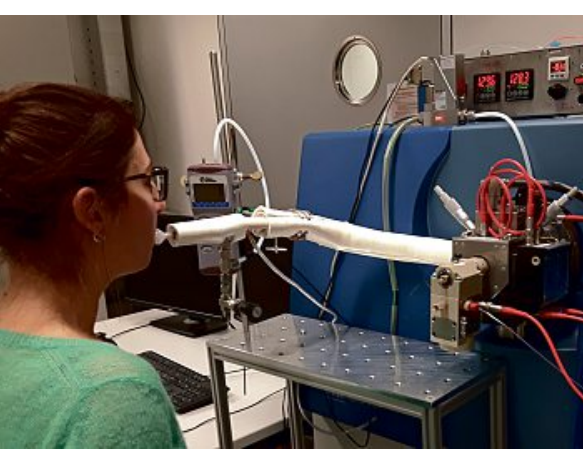

Fig. 1. Real-time exhaled breath analysis by secondary electrospray ionization-mass spectrometry.

eases, characterize different phenotypes, and to take a step forward towards a more personalized medicine. This article gives an overview over our previous and current work in this field, which was mostly done in a collaboration between ETH Zurich and the University Hospital Zurich (USZ) under the umbrella of Hochschulmedizin Zürich. ${ }^{[13]}$

\section{Detection of Exhaled Metabolites Requires High-end Instrumentation}

Most exhaled metabolites occur in extremely low concentrations (parts-pertrillion), and are present as an incredibly complex mixture of exogenous and endogenous compounds. This requires a highly sensitive and specific detection method. SESI has the key advantage that it can be applied in positive and negative ion mode, expanding the scope of detectable molecules and adding a component of chemical selectivity. Additionally, it can be coupled to virtually any mass spectrometer, enabling it to make use of recent advances in analytical instrumentation. SESI-MS can therefore overcome many limitations of other on-line methods to analyze exhaled breath regarding compound coverage, sensitivity and specificity. Furthermore, the possibility to detect heavier metabolites ( $>150$ Da) strongly improves the chances to detect robust biomarkers. However, absolute quantification with SESI-MS remains a challenge to date, due to the lack of a suitable sample delivery system for compounds with such low vapor pressures.

By coupling a new, commercial SESI source to a high-resolution mass spectrometer we were able to capture hundreds of exhaled metabolites on-line, with masses up to $900 \mathrm{Da}$, including several chemical families related to smoking behavior. ${ }^{[14]}$ During this study, we could also show the importance of high-resolution in real-time breath metabolomics. Since no chromatographic separation is performed, one usually detects several isobaric compounds at any given $\mathrm{m} / \mathrm{z}$ value. Even at resolving powers over 60,000 we could observe overlapping isobaric compounds (Fig. 2).[14] This leads to a loss of information and difficulties in compound identification.

In SESI, the analyte vapors (i.e. breath) are brought into contact with the electrospray plume of a polar solvent, resulting in ionization predominantly via protontransfer reactions in positive ion mode. However, our understanding of the ionization mechanism of SESI is still limited. We therefore conducted mechanistic studies to investigate the role of gas-phase ion chemistry, and elucidate the reactant ions in the presence of humidity. We could show that protonated water clusters play a key role in the final proton-transfer step using deuterium labelling experiments. ${ }^{[15]}$ However, several aspects of the ionization mechanism remain to be clarified in further studies.

\section{Monitoring Metabolic Pathways by Breath Analysis}

In order to make metabolic interpretations, identification of the detected metabolites is crucial. Due to the lack of chromatographic separation in real-time analysis, a complementary technique is required for this purpose. We therefore use ultra-high performance liquid chromatography-mass spectrometry (UHPLCMS) of exhaled breath condensate (EBC), which allows for unambiguous identification by comparison of retention times and $\mathrm{MS}^{2}$ fragmentation spectra with commercial standards. Additionally, on-line $\mathrm{MS}^{2}$ experiments can be conducted, for further confirmation that a certain compound is indeed detected in real-time. Using this approach, a variety of metabolites were

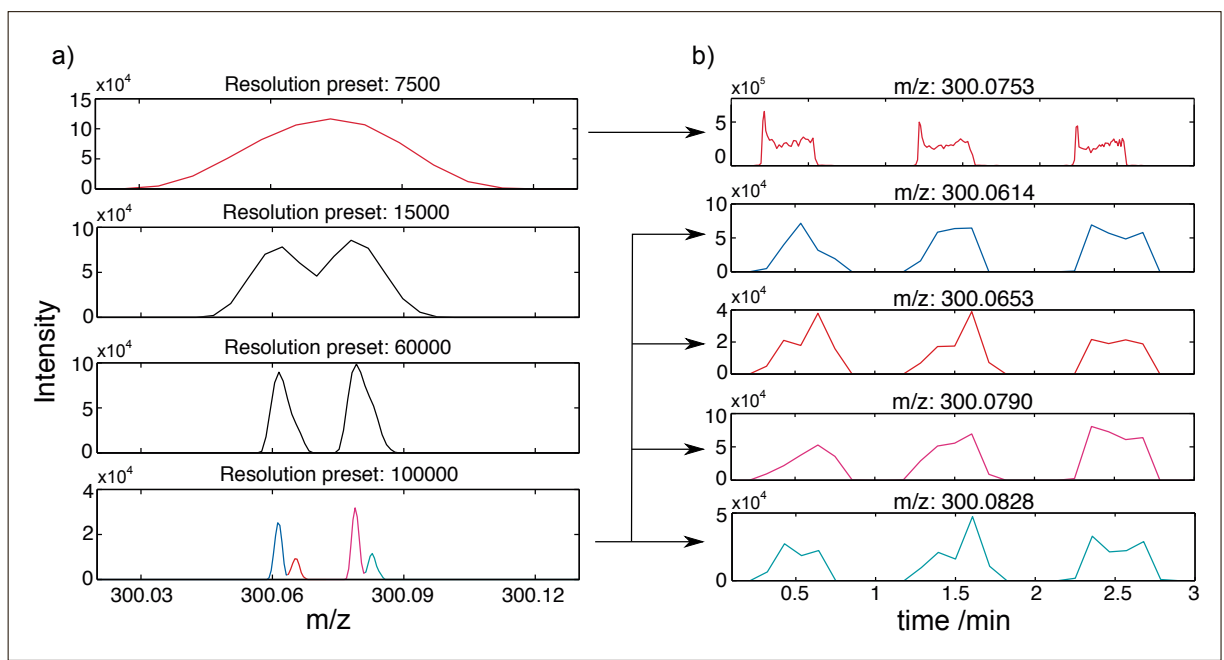

Fig. 2. Resolving power of the mass analyzer is crucial for untargeted real-time breath analysis. a) zoom of mass spectra of exhaled breath recorded at increasing resolution; b) the corresponding time traces for the top and the bottom features over three exhalations. Reproduced from ref. [14] under a CC-BY-3.0 license. identified in exhaled breath during the last years, including different aldehydes,[16] amino acids, ${ }^{[17]}$ and more. ${ }^{[18]}$ By looking at correlations of signal intensities, either within a subject or across a bigger population, it is possible to make statements about an underlying metabolic pathway. We recently could show that exhaled breath contains three full homologous series of metabolites (namely $\omega$-hydroxyalkanoic acids, $\omega$-oxoalkanoic acids and alkanedioic acids) with carbon chain lengths of 5-15 that are interconnected via the $\omega$-oxidation pathway (see Figs 3 and 4).[19]

Metabolic processes are often difficult to capture. Especially when fast conversion rates are involved, a high time resolution is required to obtain good results. Therefore, SESI-MS has been proposed as a tool to monitor pharmacokinetics and response to drugs. A study conducted in our group examined the pharmacokinetics of the anesthetic drug ketamine in mouse breath, where excellent correlations to blood levels were observed, but captured with high time resolution and in an animal friendly fashion. ${ }^{[20]}$ We also investigated the metabolic response of patients suffering from chronic obstructive pulmonary disease (COPD) and asthma to the inhalation of salbutamol (a common bronchodilator) in a placebo-controlled study including 37 COPD patients, 13 asthmatics and 48 healthy controls. ${ }^{[21]}$ We found 131 mass spectral features, comprising of several correlating chemical families, that changed significantly after inhalation of salbutamol, but not after placebo inhalation (Fig. 5). Many observations were consistent with previous studies (e.g. increase of non-esterified fatty acids) and the lipolytic effects of beta-adrenergic agonists like salbutamol. 


\section{Biomarker Discovery Using Breath Analysis}

As summarized, we have shown the capabilities of SESI-MS to elucidate metabolic pathways via breath analysis. We therefore sought to use it to identify new biomarkers for different respiratory diseases and to get a better understanding of their underlying pathophysiology.

Chronic obstructive pulmonary disease (COPD) is one of the most prevalent chronic respiratory diseases worldwide. ${ }^{[22]}$ It is characterized by a destruction of the alveoli and the collection of mucus in the bronchi. Patients suffer from a reduced lung function, bad physical fitness and impaired life quality. With the main risk factors for COPD being smoking and advanced age, it caused over 3 million deaths in 2015.[23] Nonetheless, many aspects of the pathophysiology of COPD are still poorly understood. Diagnosis remains a long process and is mostly based on lung function tests. Additionally, COPD is a heterogeneous disease whose phenotypes are only poorly understood. In a first study involving 61 subjects, we could show that COPD patients appear to have a distinct pattern of metabolites in breath that can be captured with SESI-MS.[3a] In a second study involving 22 COPD patients and 14 controls, we could predict COPD

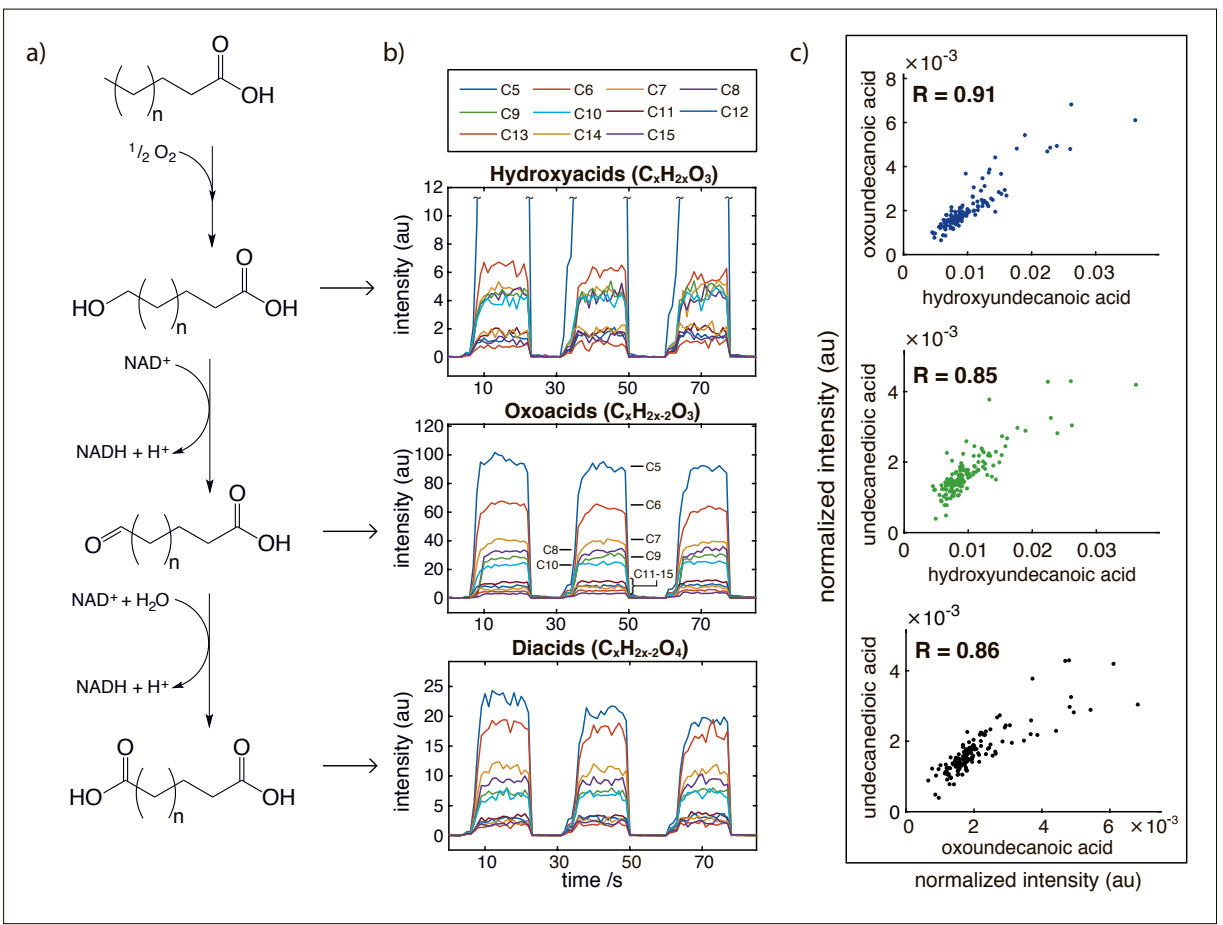

Fig. 3. On-line detection of $\omega$-oxidation metabolites in exhaled breath. a) Scheme of the $\omega$-oxidation of linear aliphatic fatty acids; b) time-traces of the corresponding metabolites detected on-line over the course of three exhalations of a healthy subject as the $[\mathrm{M}-\mathrm{H}]$. ions in negative mode; c) normalized intensities of hydroxyundecanoic acid, oxoundecanoic acid and undecanedioic acid for on-line measurements of 146 healthy subjects plotted against each other and the corresponding Pearson linear correlation coefficient. Although there might be a signal contribution of structural isomers, a strong correlation is observed. The same picture holds true for all carbon chain lengths from 5-15. Reproduced with permission from ref. [19]. Copyright (2017) American Chemical Society. a)

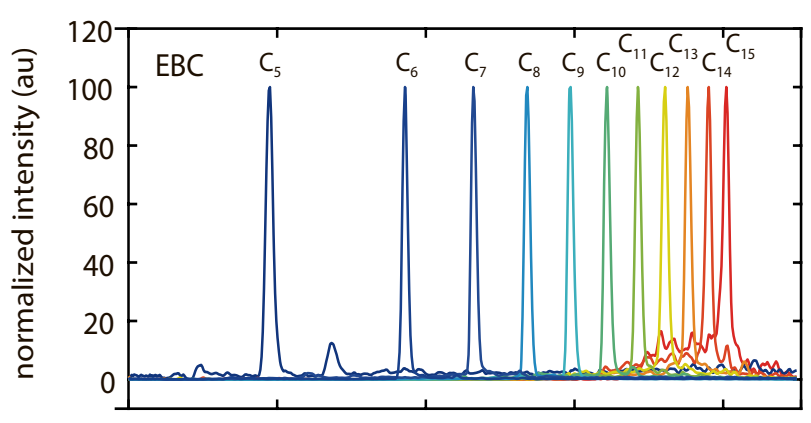

b)

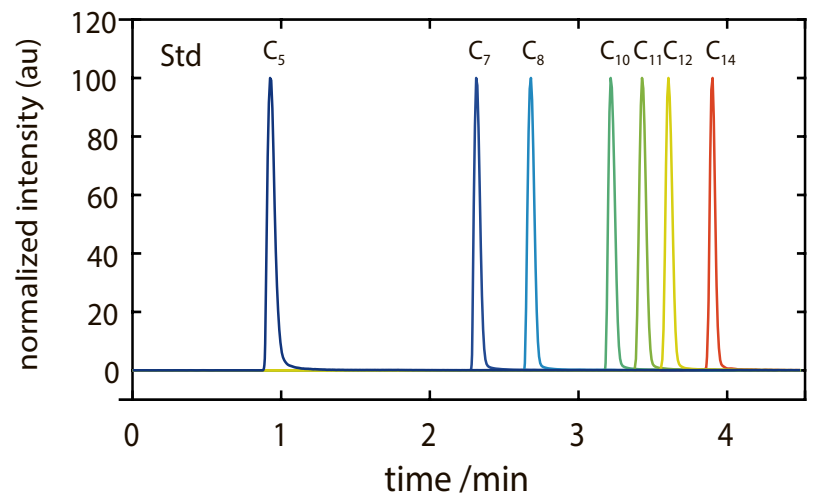

c)

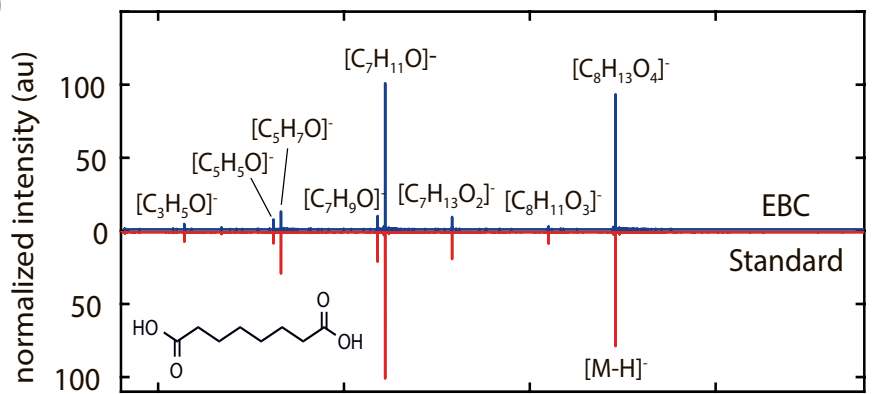

d)

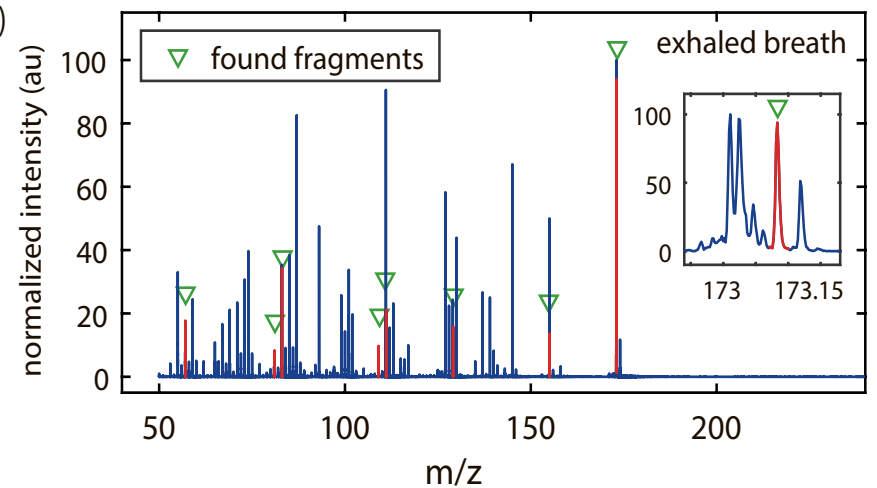

Fig. 4. Extracted ion chromatograms of the $[\mathrm{M}-\mathrm{H}]$.ions of linear, saturated dicarboxylic acids with chain lengths of 5-15 in an UHPLC-MS run of exhaled breath condensate (EBC) (a) as well as commercial standards (Std) (b). The compounds were confirmed by matching retention time and $\mathrm{MS}^{2}$ fragments with the standards. c) Head-to-tail plot of the base-peak normalized $\mathrm{MS}^{2}$ spectrum of octanedioic acid in EBC (top) and standard (bottom). d) Base-peak normalized on-line $\mathrm{MS}^{2}$ spectrum of exhaled breath (precursor: $173.1 \pm 0.35 \mathrm{Da}$ ). The insert shows a zoom of the precursor mass. As no chromatographic separation is performed, multiple parent masses are selected, explaining the abundance of other fragments. Nonetheless, the high resolution TOF analyzer makes it possible to clearly identify all fragments of octanedioic acid. Reproduced with permission from ref. [19]. Copyright (2017) American Chemical Society. 
Fig. 5. Detection of rapid response to medication by breath raw time traces for one compound $\left(\mathrm{C}_{14} \mathrm{H}_{26} \mathrm{O}_{4}\right)$ for a subject before and after inhaling placebo (a) and for another patient inhaling $200 \mu \mathrm{g}$ of salbutamol (b). The compound showed a significant relative increase 10 and 30 min after inhalation of salbutamol (d) but not after placebo (c). Reproduced from ref. [21] under a CCBY-3.0 license. analysis; exemplary

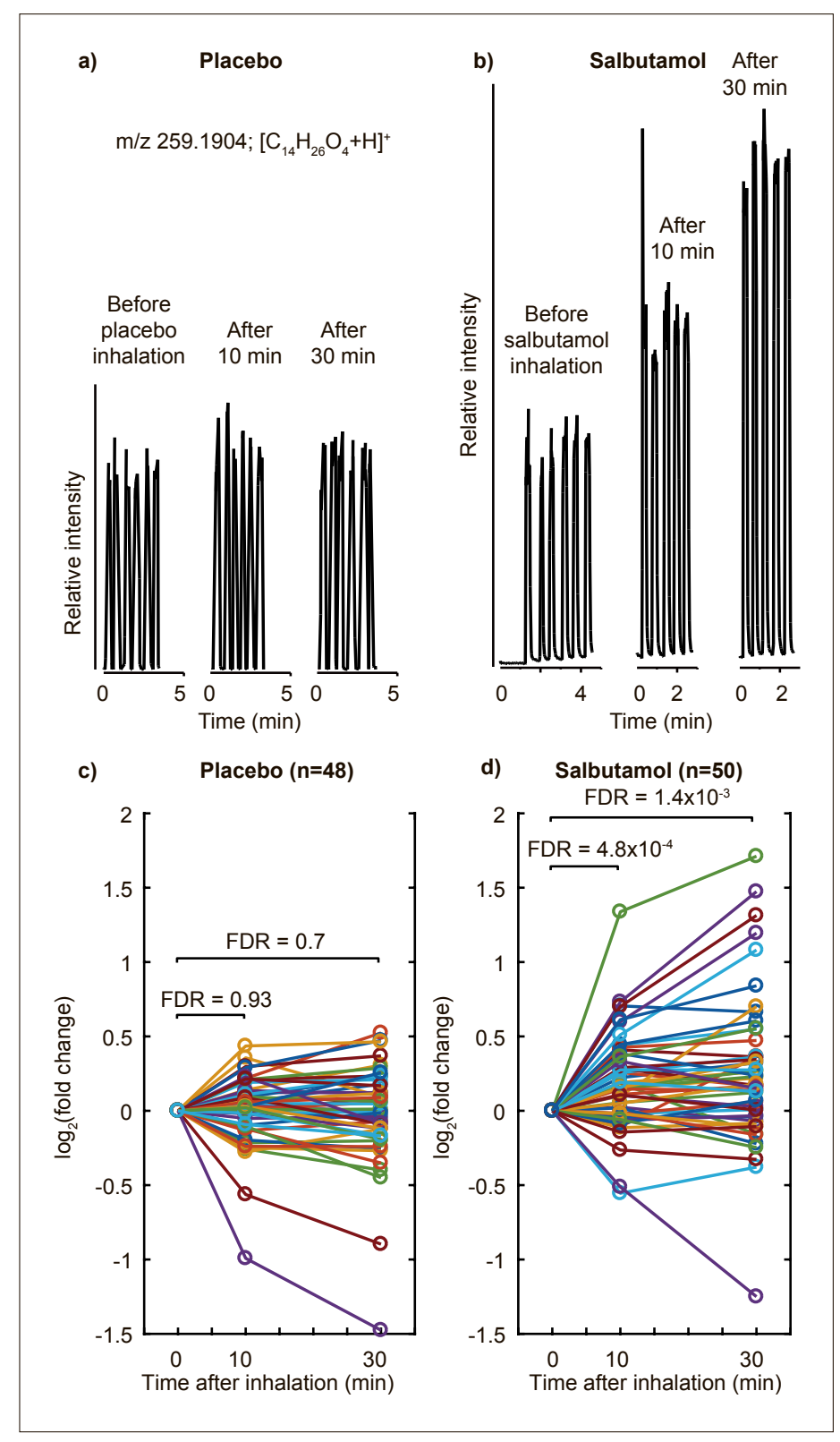

with a cross-validated accuracy of $89 \%$.[24] Several of the predictive metabolites are involved in lung muscle degradation and oxidative stress processes. Additionally, we could observe correlations of metabolites with physical capacities of the lung (lung function parameters FEV and $\mathrm{FEV}_{1} / \mathrm{FVC}$ ). However, the sample size was rather small. Therefore, we currently seek to validate these results in a larger cohort including COPD patients, asthmatics and healthy controls (data analysis ongoing). In another follow-up study, we investigated the breath profiles of COPD patients with different severities of the disease (frequent exacerbators vs. non-frequent exacerbators). Several fatty acid derivatives as well as other chemical families were highly predictive for COPD exacerbations (manuscript in preparation).

Obstructive sleep apnea (OSA) is a sleep-related breathing disorder that is characterized by intermittent breathing pauses during sleep. These are thought to cause increased oxidative stress and increased sympathetic activity by hypoxia. OSA is highly prevalent and commonly underdiagnosed as the gold standard for diagnosis is in-laboratory polysomnography, where the patient has to spend one (or several) nights at the hospital. Treatment of OSA is achieved using continuous positive airway pressure (CPAP) therapy, which effectively prevents breathing pauses, eliminating the physiological consequences of hypoxia during sleep. We therefore hypothesized that metabolic effects of hypoxia might be reflected in the exhaled breath pattern. In a randomized controlled parallel group trial, we measured the breath of 26 OSA patients on CPAP therapy (baseline) and analyzed the changes in breath profiles after half of them received placebo therapy for 2 weeks (follow-up). ${ }^{[18]}$ Home sleep studies confirmed the reoccurrence of OSA in patients receiving placebo ther- apy, and analysis of their breath spectra revealed 62 mass spectrometric features that changed significantly between baseline and follow-up measurements (Fig. 6). Several correlating chemical families could be identified within these, including closely related aldehydes (attributed to cell membrane lipid peroxidation) and furans (thought to be secondary metabolites from the gut microbiome). Using a leaveone-out-cross-validation, we could predict reoccurrence of OSA with a sensitivity of $92.9 \%$, a specificity of $84.6 \%$ and an area under the receiver operating characteristic curve of 0.87 .

\section{Outlook}

Several studies are ongoing to validate our previous results and to characterize more diseases. We are currently conducting a blind validation study for the diagnosis of OSA involving 150 subjects. Additionally, we seek to expand our results to other respiratory diseases, including asthma, idiopathic pulmonary fibrosis, and lung cancer.

Lung cancer, one of the highest impact lung diseases, is an aggressive disease and the most commonly diagnosed cancer worldwide (12.7\% of all cancers). However, diagnostic tests for lung cancer (e.g. chest CT, lung biopsies, etc.) are often only performed when symptoms are already apparent, which usually implies a very late tumor stage. As survival rates for lung cancer are highly correlated with an early diagnosis, a quick and non-invasive screening test would be a tremendous improvement. We are therefore conducting a first study on non-small cell lung cancer with the hope to take a step towards a faster diagnosis, which would have tremendous impact on mortality and patient outcome.

Lastly, instrumental improvements will be sought in order to further enhance the sensitivity and specificity of SESI-MS. Our lab is currently working on improving the standardization of breath-measurements to bring them closer towards applied clinical practice.

\section{Conclusions}

During the last years, we could show in multiple studies that on-line breath analysis with secondary electrospray ionization-mass spectrometry is a valuable tool to obtain metabolic information in a fast, non-invasive and painless fashion. Several promising biomarkers could be identified for a variety of respiratory diseases, while simultaneously obtaining new insights into the underlying pathophysiology.

Received: January 25, 2018 
[1] a) WHO, Chronic respiratory diseases, http://www.who.int/respiratory/en/, accessed 11.12.2017; b) WHO, 'The top 10 causes of death - fact sheet', http://www.who.int/ mediacentre/factsheets/fs310/en/, accessed 11.12.2017.

[2] T. H. Risby, S. F. Solga, Appl. Phys. B: Lasers and Optics 2006, 85, 421 .

[3] a) P. Martinez-Lozano Sinues, L. Meier, C. Berchtold, M. Ivanov, N. Sievi, G. Camen, M. Kohler, R. Zenobi, Respiration 2014, 87, 301; b) P. Martinez-Lozano Sinues, R. Zenobi, M. Kohler, Chest 2013, 144, 746.
[4] P. Martinez-Lozano Sinues, L. Tarokh, X. Li, M. Kohler, S. A. Brown, R. Zenobi, R. Dallmann, PLOS ONE 2014, 9, e114422.

[5] P. Martinez-Lozano Sinues, M. Kohler, R. Zenobi, PLOS ONE 2013, 8, e59909.

[6] L. Pauling, A. B. Robinson, R. Teranishi, P. Cary, Proc. Natl. Acad. Sci. USA 1971, 68, 2374.

[7] B. de Lacy Costello, A. Amann, H. Al-Kateb, C. Flynn, W. Filipiak, T. Khalid, D. Osborne, N. Ratcliffe, J. Breath Res. 2014, 8, 014001.

[8] E. Moser, M. McCulloch, J. Veterinary Behavior: Clin. Appl. Res. 2010, 5, 145.

Fig. 6. Pentenal in exhaled breath of OSA patients. Realtime breath analysis showing time traces of pentenal in a subject from the therapeutic CPAP and a subject from the CPAP-withdrawal group at baseline and at follow-up (A-D). Only in case of the patient undergoing CPAP withdrawal a strong signal increase at follow-up (4.5-fold increase) is visible. Individual plots $(E)$ of 2-pentenal in both groups $(n=26)$ show significant breath compound enhancement. Correlation (F) is shown between changes in breath signal intensity of pentenal and changes in oxygen desaturation index (ODI).

Reproduced from ref. [18] with permission from BMJ Publishing Group Ltd.

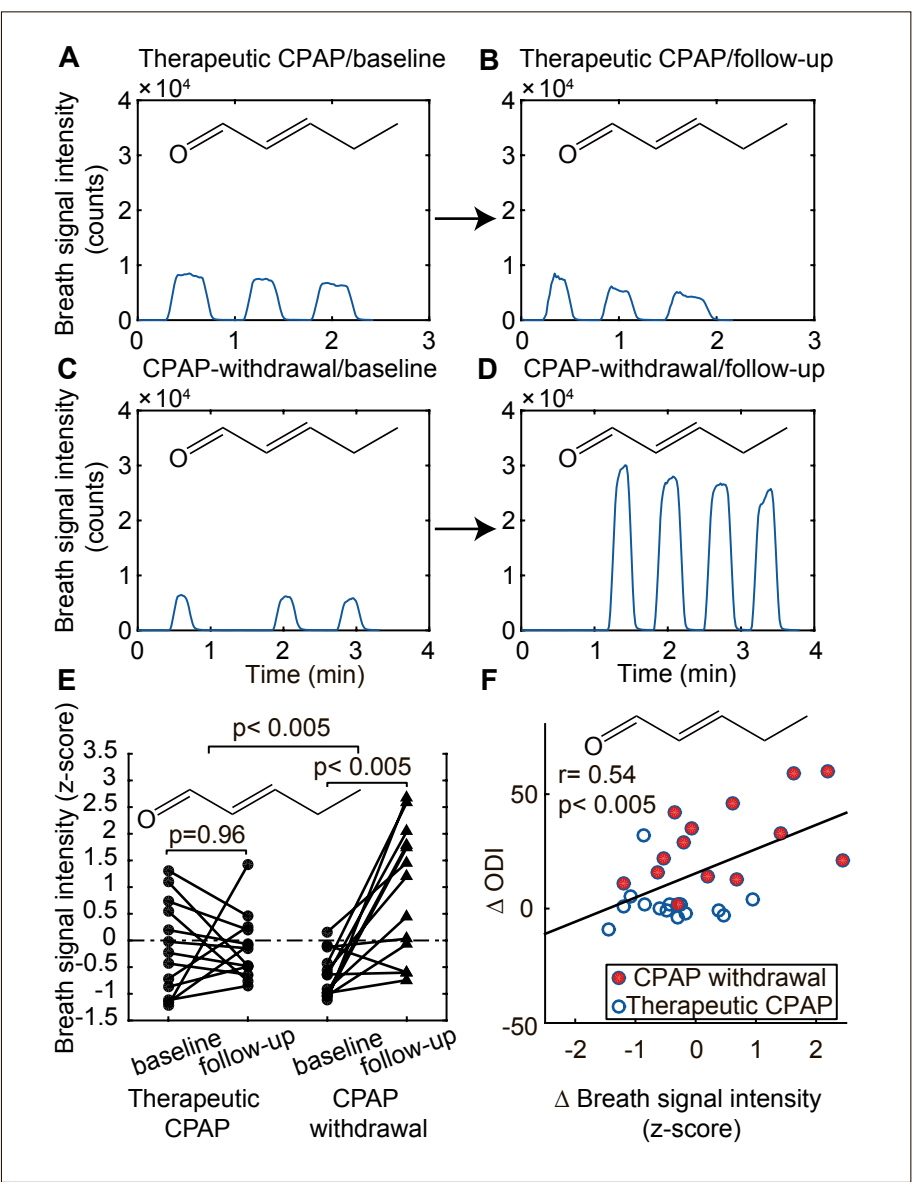

[9] P. Y. Yin, R. Lehmann, G. W. Xu, Anal. Bioanal. Chem. 2015, 407, 4879.

[10] J. Herbig, M. Müller, S. Schallhart, T. Titzmann, M. Graus, A. Hansel, J. Breath. Res. 2009, 3, 027004.

[11] P. Španěl, D. Smith, Mass Spectrom. Rev. 2011, 30, 236.

[12] P. Martínez-Lozano, J. Fernández de la Mora Int. J. Mass Spectrom. 2007, 265, 68.

[13] Zürich Exhalomics, http://www. hochschulmedizin.uzh.ch/de/projekte/zurichexhalomics.html, accessed 11.12.2017.

[14] M. T. Gaugg, D. G. Gomez, C. Barrios-Collado, G. Vidal-de-Miguel, M. Kohler, R. Zenobi, P. Martinez-Lozano Sinues, J. Breath Res. 2016, 10, 016010 .

[15] A. T. Rioseras, M. T. Gaugg, P. M. L. Sinues, Anal. Meth. 2017, 9, 5052.

[16] D. García-Gómez, P. Martínez-Lozano Sinues, C. Barrios-Collado, G. Vidal-De-Miguel, M. Gaugg, R. Zenobi, Anal. Chem. 2015, 87, 3087.

[17] D. Garcia-Gomez, T. Gaisl, L. Bregy, A. Cremonesi, P. M. Sinues, M. Kohler, R. Zenobi, Clin. Chem. 2016, 62, 1230.

[18] E. I. Schwarz, P. M. L. Sinues, L. Bregy, T. Gaisl, D. G. Gomez, M. T. Gaugg, Y. Suter, N. Stebler, Y. Nussbaumer-Ochsner, K. E. Bloch, J. R. Stradling, R. Zenobi, M. Kohler, Thorax 2016, 71, 110.

[19] M. T. Gaugg, T. Bruderer, N. Nowak, L. Eiffert, P. M. L. Sinues, M. Kohler, R. Zenobi, Anal. Chem. 2017, 89, 10329.

[20] X. Li, P. Martinez-Lozano Sinues, R. Dallmann, L. Bregy, M. Hollmen, S. Proulx, S. A. Brown, M. Detmar, M. Kohler, R. Zenobi, Angew. Chem. Int. Ed. 2015, 54, 7815.

[21] M. T. Gaugg, A. Engler, Y. NussbaumerOchsner, L. Bregy, A. S. Stoberl, T. Gaisl, T. Bruderer, R. Zenobi, M. Kohler, P. M. L. Sinues, J. Breath. Res. 2017, 11, 046004.

[22] D. Adeloye, S. Chua, C. W. Lee, C. Basquill, A. Papana, E. Theodoratou, H. Nair, D. Gasevic, D. Sridhar, H. Campbell, K. Y. Chan, A. Sheikh, I. Rudan, Gherg, J. Glob. Health 2015, 5, 186.

[23] WHO, 'Chronic obstructive pulmonry disease (COPD) - Fact sheet', http://www.who.int/ mediacentrelfactsheets/fs315/en/, accessed 05.12.2017.

[24] L. Bregy, Y. Nussbaumer-Ochsner, P. MartinzLozano Sinues, D. Garcia-Gomez, Y. Suter, T. Gaisl, N. Stebler, M. T. Gaugg, M. Kohler, R. Zenobi, Clin. Mass Spectrom. 2018, 7, 29. 